\title{
Diagnosis of pneumococcal pneumonia by polymerase chain reaction (PCR) in whole blood: a prospective clinical study
}

\author{
M L L Lorente, M Falguera, A Nogués, A Ruiz González, M T Merino, \\ M Rubio Caballero
}

\begin{abstract}
Background-Streptococcus pneumoniae is the leading cause of community acquired pneumonia; however, only a small proportion of cases can be detected by conventional methods. The ability of the polymerase chain reaction (PCR) test performed on whole blood samples to identify patients with pneumococcal pneumonia was investigated.

Methods-One hundred and fourteen consecutive adult patients with community acquired pneumonia were evaluated by a wide battery of diagnostic tests in order to determine the aetiology. Blood samples from these patients and 50 controls were also tested by the nested PCR test to detect selected pneumolysin gene fragments of $S$ pneumoniae.

Results-The patients were divided into four groups: (1) 40 patients with pneumococcal pneumonia in 22 of whom (55\%) the PCR was positive (eight of 11 with bacteraemia and 14 of 29 without); (2) 30 with pneumonia due to other pathogens in all of whom the PCR was negative; (3) 44 with pneumonia of unknown aetiology in 14 of whom (32\%) PCR was positive, and (4) 50 controls in whom the PCR test was positive in two (4\%). Thus, the sensitivity of the test was $55 \%$ and the specificity $100 \%$ (81\% if positive PCR tests among undiagnosed patients are considered as false positive results).

Conclusion-PCR applied to whole blood samples appears to be a sensitive and very specific diagnostic test for identifying patients with pneumococcal pneumonia with a potential application in clinical practice.

(Thorax 2000;55:133-137)
\end{abstract}

Keywords: pneumococcal pneumonia; polymerase chain reaction; diagnosis

A definite diagnosis of pneumococcal pneumonia using conventional methods is currently difficult to establish. The isolation of Streptococcus pneumoniae from blood or pleural fluid, the gold standard tests, is made in only $15-40 \%$ of cases, and the results obtained from sputum lack specificity. ${ }^{12}$

New methods are being evaluated to improve the diagnostic yield in patients with pneumonia. Pneumococcal antigen detection tests and serological tests have failed to show uniform diagnostic sensitivity and specificity. ${ }^{3}$ Furthermore, the indications for invasive methods to obtain the most representative samples of lung tissue remain controversial because of the risk of side effects. ${ }^{45}$

The development of the polymerase chain reaction (PCR) test holds promise as a rapid and specific test that can detect very small amounts of a specific micro-organism. ${ }^{6}$ PCR has been used in selected groups of patients to detect $S$ pneumoniae in middle ear fluid from patients with acute otitis media, ${ }^{7}$ in cerebrospinal fluid from patients with meningitis, ${ }^{8}$ in pleural fluid or transthoracic needle aspirations from patients with pneumonia, ${ }^{9}$ and in serum or blood from patients with bacteraemia. ${ }^{10-12} \mathrm{~A}$ detailed review of results obtained from these studies suggests that the PCR assay has a higher sensitivity than culture.

We have compared the usefulness of PCR with other diagnostic methods to detect the $S$ pneumoniae genome in whole blood for the diagnosis of pneumococcal pneumonia in a group of unselected patients with community acquired pneumonia.

\section{Methods}

STUDY SUBJECTS

Between September 1996 and March 1998 114 consecutive adult patients with a clinical and radiological picture suggestive of community acquired pneumonia admitted to the emergency room of a 500 bed university hospital in Catalonia (Spain) were enrolled in the study. Informed consent was obtained from all patients and the study was approved by the ethical and scientific committees of our institution. Fifty patients with a wide variety of diseases and without a recent history of pneumococcal infection were used as controls.

\section{SPECIMEN COLLECTION}

The following battery of samples was collected from patients with community acquired pneumonia:

(1) two blood samples for aerobic and anaerobic conventional cultures;

(2) an additional blood sample for study by nested PCR was obtained and stored at $-70^{\circ} \mathrm{C}$ and processed at the end of study; this sample was also collected from controls;

(3) when present, pleural fluid was biochemically examined and processed for aerobic and anaerobic cultures and, in some cases, for detection of $S$ pneumoniae genome by nested PCR; 
(4) considerable effort was made to obtain a good sputum sample; this was microscopically assessed to confirm its quality and, if satisfactory, it was cultured by conventional methods. Sputum was also stained and cultured for Mycobacterium spp or opportunistic pathogens only when it was indicated;

(5) after exclusion of contraindications, transthoracic needle aspiration (TNA) was performed in patients with severe pneumonia by the previously described technique. ${ }^{13}$ The sample obtained was processed for culture in aerobic and anaerobic media and for nested PCR to detect $S$ pneumoniae genome;

(6) based on clinical suspicion, a urine sample was obtained from some patients for detection of Legionella pneumophila antigen;

(7) a serum sample was obtained for serological investigations and stored at $-70^{\circ} \mathrm{C}$. After 4-6 weeks of follow up a second serum sample was collected and processed with the first one to detect antibodies to Mycoplasma pneumoniae (immunofluorescence test), Chlamydia pneumoniae (microimmunofluorescence test), Coxiella burnetii (complement fixation test), and $L$ pneumophila (immunofluorescence test).

PCR TECHNIQUE

$200 \mu \mathrm{l}$ samples of whole blood from patients with community acquired pneumonia and control subjects or $200 \mu \mathrm{l}$ specimens obtained by TNA or thoracocentesis were processed using the QIAamp Blood Kit for extraction of $S$ pneumoniae DNA. Proteinase $\mathrm{K}$ is used in this method for DNA extraction.

The selection of two primer pairs was based on the published pneumolysin gene sequence ${ }^{14}$; the outer primers Ia (5'-ATTTCTGTAACA GCTACCAACGA-3') and Ib (GAATTCC CTGTCTTTTCAAAGTC-3) amplified a $348 \mathrm{bp}$ region and the inner primers IIa (5'-CCCACTTCTTCTTGCGGTTGA-3') and IIb (5'-TGAGCCGTTATTTTTTCAT ACTG-3') amplified a $208 \mathrm{bp}$ region of the pneumolysin gene.

A mixture was prepared using the following reagents: $10 \mu \mathrm{l}$ PCR buffer (200 $\mathrm{mM}$ Tris-ClH $\mathrm{pH} 8.4,500 \mathrm{mM} \mathrm{ClK}), 3 \mu \mathrm{l} 50 \mathrm{mM} \mathrm{Cl}{ }_{2} \mathrm{Mg}$, $1 \mu 110 \mathrm{mM}$ dNTP mixture, $1 \mu \mathrm{l}$ of the first primer pair (Ia and Ib) (100 pmol for each reaction), 2.5 Taq polymerase units, made up to $50 \mu \mathrm{l}$ with distilled water. In a $1.5 \mathrm{ml}$ tube $100 \mu \mathrm{l}$ of mineral oil, $50 \mu \mathrm{l}$ of mixture $2 \mathrm{X}$, and $50 \mu \mathrm{l}$ of the study sample were instilled and the following thermocycle programme was performed (Mod IHB2024, Cherlyn Electronics Ltd, Cambridge, UK): one cycle of four minutes at $95^{\circ} \mathrm{C} ; 30$ cycles of one minute at $95^{\circ} \mathrm{C}$, one minute at $55^{\circ} \mathrm{C}$, and one minute at $72^{\circ} \mathrm{C}$ : and one cycle of 10 minutes at $72^{\circ} \mathrm{C}$. The same operation was repeated with the second pair of primers (IIa and IIb).

The amplified product was analysed by $2 \%$ agarose gel electrophoresis and ethidium bromide staining and examined by ultraviolet transillumination. Identification of the size of the band was performed by comparison with standard molecular weights (100 base pairs DNA ladder; Gibco BRL) and the band obtained from positive controls.

To prevent contamination a strict spatial separation of different PCR steps was maintained during the process and the recommendations of Kwok and Higuchi were followed. ${ }^{15}$ In addition, a positive control formed by a suspension of pneumococci in distilled water and a negative control for each sample consisting of target free distilled water were included in the procedure. Sensitivities of the assay on TNA and blood samples have previously been reported. ${ }^{9} 10$

AETIOLOGICAL CLASSIFICATION OF PATIENTS

According to the microbiological results and excluding PCR results provided by blood samples, the patients were classified into four groups:

(1) Patients with pneumococcal pneumonia: a definite diagnosis of pneumococcal pneumonia was established when $S$ pneumoniae was isolated from an uncontaminated sample (blood, pleural fluid, or lung aspirate) or the $S$ pneumoniae genome was detected in pleural fluid or TNA sample. In contrast, a probable diagnosis was made when $S$ pneumoniae was only isolated in a pure culture from the sputum. Mixed infections, including $S$ pneumoniae in the isolated flora, were also considered as pneumococcal pneumonias.

(2) Patients with pneumonia due to other aetiologies: a fourfold or greater rise in serological titres, the isolation of a microorganism from uncontaminated samples (blood, pleural fluid, or TNA) or, for obligate pathogens, from the sputum, as well as the detection of $L$ pneumophila antigen in urine, constituted a definite aetiological diagnosis. Conventional pathogens isolated only from the sputum were considered as a probable microbial diagnosis. Some patients finally diagnosed as having tuberculosis were included in this group.

(3) Patients with pneumonia of unknown aetiology: all the remaining patients with nondiagnostic microbiological results were classified into this group.

(4) Patients with other pathologies were used as controls.

STATISTICAL ANALYSIS

The sensitivity and specificity of PCR for $S$ pneumoniae in the blood were calculated by comparison with the results provided by other diagnostic methods.

\section{Results}

PATIENT CHARACTERISTICS

A total of 164 patients, 114 with community acquired pneumonia and 50 controls, were enrolled in the study. The mean age of the patients was 62 years (range 18-94); 69 (61\%) were men and $70(61 \%)$ had coexisting diseases. Before admission 54 (47\%) had received antimicrobial therapy. The initial chest radiograph showed segmental, lobar, or 
Table 1 Aetiology of community acquired pneumonia in 114 studied patients

\begin{tabular}{llll}
\hline Micro-organisms & $\begin{array}{l}\text { Definite } \\
\text { diagnosis }\end{array}$ & $\begin{array}{l}\text { Probable } \\
\text { diagnosis }\end{array}$ & Total \\
\hline Streptococcus pneumoniae & 38 & 2 & $40^{\star}(35 \%)$ \\
Other pathogens: & 26 & 4 & $30(26 \%)$ \\
$\quad$ Mycoplasma pneumoniae & 11 & 0 & $11(10 \%)$ \\
Mycobacterium tuberculosis & 4 & 0 & $4(4 \%)$ \\
Staphylococcus aureus & 2 & 1 & $3(3 \%)$ \\
Chlamydia pneumoniae & 2 & 0 & $2(2 \%)$ \\
Legionella pneumophila & 2 & 0 & $2(2 \%)$ \\
Streptococcus viridans & 2 & 0 & $2(2 \%)$ \\
Pseudomonas aeruginosa & 0 & 2 & $2(2 \%)$ \\
Coxiella burnetii & 1 & 0 & $1(1 \%)$ \\
Haemopilus influenzae & 1 & 0 & $1(1 \%)$ \\
Escherichia coli & 0 & 1 & $1(1 \%)$ \\
Cryptococcus neoformans & 1 & 0 & $1(1 \%)$ \\
Unknown aetiology & 44 & 0 & $44(39 \%)$ \\
\hline *ne patient had a dual infection & $(S$ pneumoniae $+M$ \\
pneumoniae). & & \multicolumn{3}{l}{}
\end{tabular}

Table 2 Patients with pneumococcal pneumonia: results of diagnostic techniques

\begin{tabular}{ll}
\hline Technique & Sensitivity \\
\hline Blood culture & $11 / 40(28 \%)$ \\
TNA culture & $6 / 24(25 \%)$ \\
Pleural fluid culture & $6 / 10(60 \%)$ \\
Sputum culture & $5 / 25(20 \%)$ \\
Pleural fluid PCR & $2 / 4(50 \%)$ \\
TNA PCR & $16 / 22(73 \%)$ \\
Blood PCR & $22 / 40(55 \%)$ \\
\hline
\end{tabular}

TNA $=$ transthoracic needle aspiration; $\mathrm{PCR}=$ polymerase chain reaction.

multilobar consolidation in all cases, and pleural effusion was detected in $16(14 \%)$. After initial evaluation 94 patients required admission to hospital while 19 were managed as outpatients. During follow up five $(4 \%)$ required admission to the intensive care unit and nine (8\%) died.

The control subjects had a mean age of 68 years (range 25-91); 28 (56\%) were men and the reasons for attendance were cardiac disease (14), chronic obstructive pulmonary disease (12), cerebrovascular disease (9), neoplasms (6), other (9).

\section{AETIOLOGICAL STUDIES}

Blood samples for culture, PCR, and the first serological determination were obtained from all patients. The blood cultures were positive in

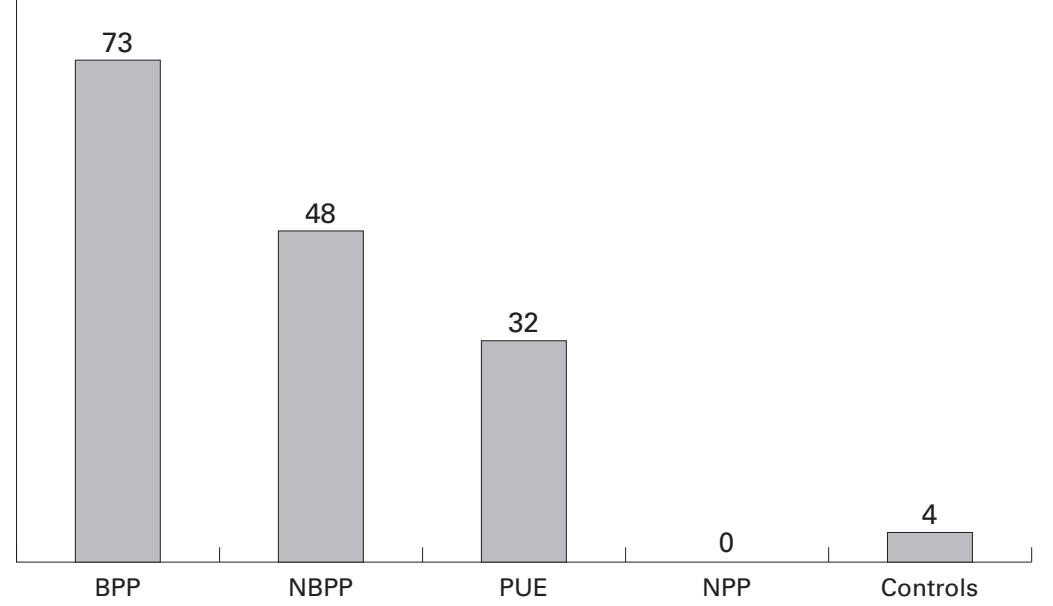

Figure 1 Percentage of positive PCR results in blood among different subgroups of patients. $B P P=$ bacteraemic pneumococcal pneumonia $(n=11) ; N B P P=$ non-bacteraemic pneumococcal pneumonia $(n=29) ; P U E=$ pneumonia of unknown aetiology $(n=44)$; $N P P=$ non-pneumococcal pneumonia $(n=30)$; controls = patients without pneumonia $(n$ $=50)$.
$17(15 \%)$, yielding $S$ pneumoniae in 11 . The second serological sample was collected from 56 patients $(49 \%)$ and a diagnosis was obtained in 17 (M pneumoniae 12, C pneumoniae 2, L pneumophila 2 , and $C$ burnetii 1$)$.

TNA was performed in 47 patients (41\%). Cultures isolated $S$ pneumoniae in six patients and PCR was positive in 16. Moreover, cultures were also positive for additional pathogens in four cases.

Gram staining and culture of sputum was performed in 62 patients $(54 \%)$ and $S$ pneumoniae was isolated from five of them. In addition, four cases of tuberculosis were detected by means of adequate staining and/or culture of sputum. Finally, pleural fluid recovered from 16 patients $(14 \%)$ showed the presence of $S$ pneumoniae by culture or PCR in eight patients.

Thus, a bacteriological diagnosis was made in 70 cases $(61 \%$, table 1$)$, distributed as follows. Pneumococcal pneumonia was diagnosed in 40 patients, establishing a definite diagnosis in 38 and a probable diagnosis in two. One patient who had a mixed infection ( $S$ pneumoniae plus $M$ pneumoniae) was included in this group. The results of different diagnostic techniques are shown in table 2. The diagnosis of non-pneumococcal pneumonia was made in 30 cases. $M$ pneumoniae was the most frequent micro-organism in this group. Table 1 shows the distribution of pathogens. A definite diagnosis was established in 26 cases and a probable diagnosis in four. Finally, in 44 patients no definite microbial diagnosis was made.

RESULTS OF PCR IN WHOLE BLOOD FOR $S$ PNEUMONIAE DETECTION

PCR was positive in 22 of 40 patients with pneumococcal pneumonia yielding a sensitivity of $55 \%$ (95\% CI 40 to 70 ). As shown in fig 1, patients with bacteraemia had a higher rate of positive PCR results than non-bacteraemic cases $(73 \%$ versus $48 \%)$, although the difference was not statistically significant. If the diagnosis of pneumococcal pneumonia was only based on culture results, excluding cases detected by PCR on TNA or pleural fluid samples, the sensitivity of PCR in blood improved. Thus, among 24 culture proven cases PCR in blood was positive in 14 (sensitivity $58 \% ; 95 \%$ CI 38 to 78 ). Furthermore, PCR in whole blood was negative in all 30 patients with nonpneumococcal pneumonia, giving a specificity of $100 \%$. On the other hand, in 44 patients with pneumonia of unknown aetiology the PCR test was positive in $14(32 \%)$; if these are considered as false positive results the calculated specificity would be $81 \%$ (95\% CI 71 to $89)$. Finally, the test was positive in two of the 50 controls (4\%), both of whom were stable patients with chronic obstructive pulmonary disease.

\section{Discussion}

In this study the PCR technique was routinely performed to detect $S$ pneumoniae DNA in whole blood from patients with community acquired pneumonia. The results show that, in comparison with an extensive battery of 
alternative diagnostic methods, nested PCR in whole blood was one of the most sensitive $(55 \%)$ and a very specific $(100 \%)$ test, with a potential practical value in the clinical setting.

$S$ pneumoniae is the leading cause of community acquired pneumonia in adults worldwide; however, studies have found wide differences in its relative incidence rate with values ranging from $6 \%$ to $76 \%$ among similar populations. ${ }^{16}{ }^{17}$ In addition, the responsible pathogen is usually not found in about $50 \%$ of cases; for many investigators most of these undetermined cases may be due to $S$ pneumoniae. ${ }^{18}{ }^{19}$ We can therefore conclude that the diagnosis of pneumococcal pneumonia remains an unresolved problem.

The use of PCR of blood samples for the detection of $S$ pneumoniae DNA in certain subgroups of patients with pneumonia has been previously studied; most of the studies included only a small number of patients with pneumococcal bacteraemia and reported sensitivities between $37 \%$ and $100 \% .^{10-12} 20-22 \mathrm{~Pa}-$ tients with pneumonia of unknown aetiology have also been evaluated by some of these investigators who found positive PCR results in $16-45 \%$ of patients, ${ }^{1120} 21$ suggesting that the test may be useful in non-bacteraemic patients. Our study has several important advantages compared with these earlier trials. We have evaluated an extensive and unselected group of patients with community acquired pneumonia and a significant effort was made to diagnose a high proportion of cases. We were therefore able to include in the analysis many well documented patients with non-bacteraemic pneumococcal pneumonia as well as many well documented patients with non-pneumococcal pneumonia corresponding with the habitual flora causing community acquired lower tract infections.

We found that the sensitivity of PCR in the blood for diagnosing pneumococcal pneumonia largely exceeded that obtained by conventional methods. Blood cultures are usually positive in $15-40 \%$ of cases ${ }^{1}$ so our result of $28 \%$ suggests that PCR is about twice as sensitive as other methods. Gram staining or culture of sputum have achieved acceptable levels of sensitivity and specificity in some well designed prospective trials; in retrospective observations the levels were reduced. ${ }^{323} 24$ Pleural fluid is only available in about $25 \%$ of cases, but the sensitivity of PCR on samples obtained directly from the lung parenchyma by TNA was higher, as expected; undoubtedly, lung aspiration should be the most sensitive sample in the aetiological study of pneumonia. Compared with all these alternative diagnostic tests, PCR in blood constitutes a non-invasive technique with no risks or contraindications; a good quality specimen is available from all patients, it is simple to obtain, and provides a rapid result.

In patients with pneumonia of unknown aetiology we found that $32 \%$ of cases also had a positive PCR test in blood. The absence of a sensitive gold standard test does not allow us to know the exact significance of these results. However, although we cannot exclude some false positive results, the high specificity observed among other groups of patients makes us suspect that they mainly constitute true positive cases. In concordance with previous authors we therefore suggest that a high proportion of pneumococcal infections are present among patients with pneumonia of unknown aetiology. ${ }^{18} 19$

We used the PCR technique on pleural fluid and, in particular, lung aspirates to diagnose pneumococcal pneumonia. Certainly, we would consider them as insufficiently evaluated techniques for use as reference methods. We have recently reported the value of PCR in TNA samples in concordance with culture and the pneumococcal antigen detection test. ${ }^{9}$ However, the exclusion of these PCR diagnosed cases does not significantly modify the results of PCR in blood; the sensitivity is mildly increased and the specificity is unchanged.

These results generate some important questions. Why did some controls give positive PCR results and, conversely, why did some bacteraemic patients give negative PCR results? PCR is still an imperfect method. Previous studies have also obtained positive results in control patients. ${ }^{102021}$ Some investigators have related the false positive results of the assay to nasopharyngeal carriage of $S$ pneumoniae which, being children, is the group with the highest rate of false positive results ${ }^{20}$; the same explanation could be given for other groups of patients with a high incidence of pneumococcal colonisation of the airways. In addition, the procedure is subject to contamination with exogenous material, particularly in the laboratory, so the introduction of negative controls into the procedure is inexcusable. ${ }^{6} \mathrm{On}$ the other hand, inhibitors can be present in clinical specimens, particularly in blood samples, leading to false negative results. ${ }^{25}$ Furthermore, the methodology of PCR has not yet been fully standardised and differences exist on essential aspects including the choice of primers and genes for amplification and the methods for nucleic acid extraction, amplification, and detection.

In summary, we conclude that PCR applied to whole blood samples is already a useful tool for the diagnosis of pneumococcal pneumonia, even in non-bacteraemic patients, with a good sensitivity and high specificity. Future technical refinements will probably eliminate some imperfections of the method.

1 Mandell LA. Community-acquired pneumonia. Etiology, epidemiology, and treatment. Chest 1995;108:35-42S.

2 Reimer LG, Carroll KC. Role of the microbiology laboratory in the diagnosis of lower respiratory tract infeclaboratory in the diagnosis of lower rest

3 Skarrett SJ. Diagnostic testing to establish a microbial cause is helpful in the management of community-acquired pneumonia. Semin Respir Inf 1997;12:308-21.

4 Baughman RP, Conrado CE. Diagnosis of lower respiratory tract infections. What we have and what would be nice. Chest 1998;113:219-23S.

5 Dorca J, Manresa F, Esteban L, et al. Efficacy, safety, and therapeutic relevance of transthoracic aspiration with ultrathin needle in nonventilated nosocomial pneumonia. Am 7 Respir Crit Care Med 1995;151:1491-6.

6 Ieven M, Goossens H. Relevance of nucleic acid amplification techniques for diagnosis of respiratory tract infections tion techniques for diagnosis of respiratory tract infections
in the clinical laboratory. Clin Microbiol Rev 1997;10:24256.

7 Liederman EM, Post JC, Aul JJ, et al. Analysis of adult otitis media: polymerase chain reaction versus culture for bacteria and viruses. Ann Otol Rhinol Laryngol 1998;107:10-16. 
8 Isaacman DJ, Zhang Y, Rydquist-White J, et al. Identification of a patient with Streptococcus pneumoniae bacteremi and meningitis by the polymerase chain reaction (PCR) Mol Cell Prob 1995;9:157-60.

9 Ruiz-González A, Nogués A, Falguera M, et al. Rapid detection of pneumococcal antigen in lung aspirates: comparison with culture and PCR technique. Respir Med 1997;91: 201-6.

10 Salo P, Örtqvist A, Leinonen M. Diagnosis of bacteremic pneumococcal pneumonia by amplification of pneumolysin gene fragment in serum. F Infect Dis 1995;171:479-82.

11 Zhang Y, Isaacman DJ, Wadowsky RM, et al. Detection of Streptococcus pneumoniae in whole blood by PCR. 7 Clin Streptococcus pneumoniae in
Microbiol 1995;33:596-601.

12 Rudolph KM, Parkinson AJ, Black CM, et al. Evaluation of polymerase chain reaction for diagnosis of pneumococcal pneumonia flin Microbiol 193,31:2661-6.

13 Falguera M, Nogués A, Ruiz-González A, et al. Transthoracic needle aspiration in the study of pulmonary infection in patients with HIV. Chest 1994;106:697-702.

14 Walker JA, Allen RL, Falmagne P, et al. Molecular cloning, characterization, and complete nucleotide sequence of the gene for pneumolysin, the sulfhydryl-activated toxin of Streptococcus pneumoniae. Infect Immun 1987;55:1184-9.

15 Kwok SK, Higuchi R. Avoiding false positives with PCR. Nature 1989;339:237-8.

16 Bates JH, Campbell GD, Barron AL, et al. Microbial etiology of acute pneumonia in hospitalized patients. Chest

17 Macfarlane JT, Finch RG, Ward MJ, et al. Hospital study of adult community-acquired pneumonia. Lancet 1982;ii: $255-8$
18 Farr BM, Kaiser DL, Harrison BDW, et al. Prediction of microbial aetiology at admission to hospital for pneumonia from the presenting clinical features. Thorax 1989;44 1031-5.

19 Bohte R, Hermans J, van der Broek PJ. Early recognition of Streptococcus pneumoniae in patients with communityacquired pneumonia Eur $\mathcal{f}$ Clin Microbiol Infect Dis 1996;15:201-5.

20 Dagan R, Shriker O, Hazan I, et al. Prospective study to determine clinical relevance of detection on pneumococcal DNA in sera of children by PCR. 7 Clin Microbiol 1998;36: 669-73.

21 Isaacman DJ, Zhang Y, Reynolds EA, et al. Accuracy of a polymerase chain reaction-based assay for detection of pneumococcal bacteremia in children. Pediatrics 1998;101: 813-6.

22 Menéndez R, de la Cuadra P, Córdoba J, et al. Aetiology of community acquired pneumonia: clinical utility of the polymerase chain reaction in non-invasive samples. European Respiratory Society, Annual Congress 1998: 1036.

23 Watanakunakorn C, Bailey TA. Adult bacteremic pneumococcal pneumonia in a community teaching hospital, 1992-1996. A detailed analysis of 108 cases. Arch Intern Med 1997;157:1965-71.

24 Torres JM, Cardenas O, Vasquez A, et al. Streptococcus pneumoniae bacteremia in a community hospital. Chest 1998; 113:387-90.

25 Higuchi R. Simple and rapid preparation of samples for PCR. In: Erlich HS, ed. PCR technology: principles and applications for DNA amplification. New York: Stockton applications for DNA 